\title{
Prevalencia de bacterias Gram negativas en tilapias comercializadas en el cantón Milagro, Octubre - Noviembre 2013
}

\section{Gram negative bacteria study on tilapia sold in the canton Milagro, October-November 2013}

Luís Cagua-Montaño Laboratorio Clínico "Pazmiño"

Karen Rodas-Pazmiño Laboratorio Clínico "Pazmiño"

Jennifer Rodas-Pazmiño Laboratorio Clínico "Pazmiño"
Betty Pazmiño-Gómez Universidad Estatal de Milagro (Milagro - Guayas - Ecuador) bettypazmino@hotmail.com

Edgar Rodas-Neira Laboratorio Clínico "Pazmiño"

Gabriel González-Quinde Laboratorio Clínico "Pazmiño"

Andrea Rodas-Pazmiño Escuela Superior Politécnica del Litoral
Revista Cumbres Vol.4 №1

Versión impresa ISSN 1390-9541

Versión electrónica ISSN 1390-3365

http://investigacion.utmachala.edu.ec/revistas/index.php/Cumbres 


\title{
RESUMEN
}

Las enterobacterias pertenecen a la familia Enterobacteriaceae y se componen de un grupo extenso y heterogéneo de bacterias Gram negativas, ubicuas y oxidasa negativas, que se encuentran en forma abundante en el suelo, el agua y vegetación; son saprófitos del tubo digestivo, forman parte de la flora intestinal normal de humanos y animales, en el hombre ocasionan síndromes diarreicos acompañados de fiebre y septicemia. El objetivo de este estudio fue determinar la presencia de bacterias Gram negativas en tilapias comercializadas en la ciudad de Milagro. A través de un estudio realizado en los meses de octubre y noviembre del 2013, de un total de 48 muestras de tilapias tomadas de tres mercados de la ciudad de Milagro; procesadas en medios de cultivos selectivos (Agar MacConkey), tinción de Gram y pruebas bioquímicas; se identificaron las siguientes prevalencias de bacterias Gram negativas: Escherichia coli (46\%), Proteus mirabilis (4\%), Klebsiella oxitoca (4\%), identificándose por primera vez en el Ecuador estas bacterias en este hospedero, lo que constituye un peligro inminente para los consumidores, al ingerir tilapias sin la adecuada cocción y un manejo inadecuado.

Palabras clave: Enterobacterias, bacterias Gram negativas, tinción de Gram, tilapia.

\begin{abstract}
Enterobacteria belong to the Enterobacteriaceae family and are composed of an extensive and heterogeneous group of Gram-negative bacteria, ubiquitous and oxidase negative. They are abundantly found in the soil, water and vegetation; they are saprophytes of the digestive tract and are part of the normal intestinal flora of humans and animals. In men, they cause diarrheal syndromes accompanied by fever and septicemia. The objective of this study was to determine the presence of Gram-negative bacteria in tilapia marketed in Milagro city. The study was carried out between October and November 2013. A total of 48 samples of Tilapias was collected from 3 markets of Milagro; the tilapias were processed in selective culture media (MacConkey Agar), Gram staining and biochemical tests. The following prevalences of Gram-negative bacteria were identified: Escherichia coli (46\%), Proteus mirabilis (4\%), Klebsiella oxitoca (4\%). These bacteria were identified for the first time in Ecuador in this host, which constitutes an imminent danger for consumers when ingesting tilapia without proper cooking and improper handling.
\end{abstract}

Keywords: Enterobacteria, Gram-negative bacteria, Gram staining

\section{INTRODUCCIÓN}

Los alimentos funcionales son aquellos que, además de satisfacer las neceLas enterobacterias son bacilos Gram negativos, oxidasa negativa, aerobias y

\section{2}


anaerobias facultativas, fermentadoras de carbohidratos (glucosa y lactosa), de las que existen más de 30 géneros con 100 especies (Winn et al., 2008). Murray (2009) describe que son habituales comensales del intestino de los animales y en ciertos casos son causantes de patologías graves y pueden contaminar con facilidad los alimentos, en especial la carne de tilapias; estos microorganismos pueden ser transmitidos a los humanos por: la ingestión, manipulación de la carne contaminada y por heridas traumáticas que frecuentemente se presentan al estar en contacto con las evisceraciones del animal sacrificado.

Las enterobacterias más frecuentes en las tilapias que pueden transmitirse a humanos son: Escherichia coli, Klebsiella oxitoca, Citrobacter spp., Salmonella spp., Proteus spp., Vibrio spp., Enterobacter spp. Shigella, entre otras (Puerta-García, 2010; Dos Santos, 2014).

Comúnmente, las enterobacterias existentes en las tilapias se transmiten al hombre y suelen ocasionar síndromes diarreicos acompañados de fiebre y septicemia (Paredes, 2004). La citotoxicidad producida por estas enterobacterias tiene un rol importante en la patogenicidad de los hospederos susceptibles, dada por la capacidad de sintetizar distintas toxinas que provocan alteraciones letales en las células del humano (Leal, 2009).

En el departamento de Cundiamarca (Colombia), durante el año 2011, se realizó un análisis bacteriológico en 5 sitios de expendio de pescado fresco, en este estudio se determinó en bagre la presencia de Citrobacter amalonaticus y Citrobacter freundii en un 30\% de las muestras; Klebsiella oxytoca, Enterobacter cloacae, y Edwarsiella tarda en un 10\%, respectivamente. Respecto a la Tilapia roja se aisló Klepsiella oxytoca, Klepsiella ozaenae, Edwarsiella tarda, Proteus mirabilis en un 20\% y Vibrio metschnikovii en un 10\%; también se describe que estos microorganismos en elevadas cantidades pueden representar un alto riesgo para la salud pública (Corrales, 2011).

En el país existe escasa información sobre enfermedades prevalentes en el cultivo de tilapias y el riesgo de trasmisión a humanos (Garzón, 2014).

\section{MATERIALES Y MÉTODOS}

Metodología: El presente estudio es de tipo descriptivo, prospectivo y transversal, el universo consistió en 24 tilapias que fueron obtenidas de tres mercados de la ciudad de Milagro que son: Mercado "24 de Mayo", Mercado "La Dolorosa” y el Mercado "La Colón”, las mismas que fueron transportadas en fundas estériles de polietileno al centro bacteriológico "PAZMIÑO" para su respectiva investigación; que consistió en tomar una muestra de la zona braquial y otra de la buconasal, por cada animal, siguiendo las normas del Instituto de Estandarización de Laboratorio (CLSI, 2015). Luego, las muestras se sembraron en agar sangre de cordero y MacConkey, y se colocaron en estufa Memmert modelo um-400, procedencia alemana, a una temperatura de $35^{\circ} \mathrm{C}$ por 24 horas, para la recuperación de enterobacterias (CLSI, 2015; Forbes, 2009). Posteriormente, se realizó la tinción de Gram para identificar la bacteria presente en el medio de cultivo, por medio de microscopía óptica 
marca OLYMPUS, modelo CX31, procedencia USA y finalmente se efectuaron pruebas complementarias de oxidasa y bioquímica (Agar TSI, SIM, Citrato, Urea, Rojo metilo y Lisina), para la identificación de enterobacterias (Winn, et al., 2008; MacFaddin, 2004; Forbes, 2009).

\section{RESULTADOS Y DISCUSIÓN}

En la figura 1 se muestra la disección realizada la zona buco-nasal previa al muestreo y la disección branquial en la figura 2.

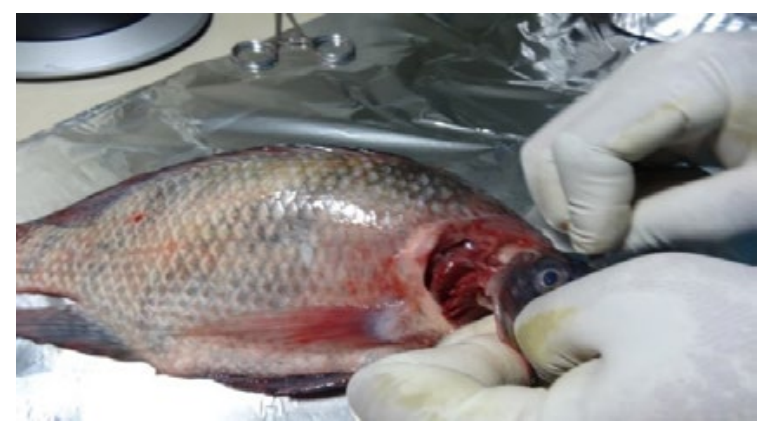

Figura 1. Disección en la zona buco-nasal, previo al muestreo.

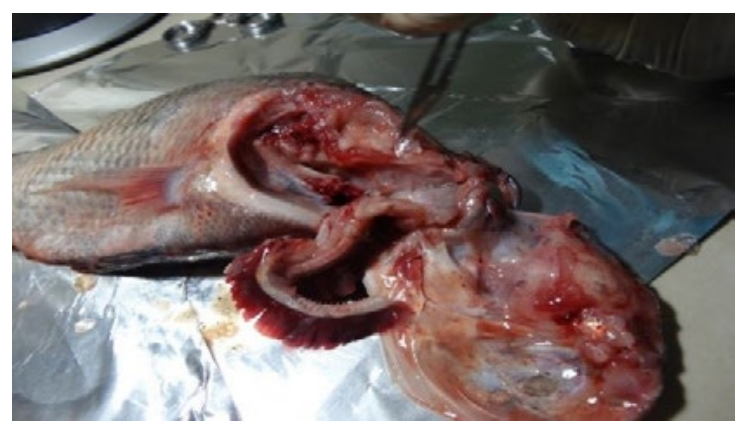

Figura 2. Disección branquial necesario para el muestreo.

Se estudiaron 48 muestras de tilapias procesadas a nivel braquial y buconasal, identificándose la siguiente prevalencia: Escherichia coli 12 muestras (25\%), Klebsiella oxitoca 1 muestra (2\%), Proteus mirabilis 1 muestra (2\%), otras bacterias 22 muestras (46\%) y negativas 12 muestras (25\%). Tabla 1.

De las 26 muestras identificadas con enterobacterias, 19 (73\%) correspondieron a la zona buconasal y 7 (27\%) de la zona branquial.

\section{$4 \quad$ Cumbres}


Tabla 1. Recuperación de enterobacterias según el sitio de toma de muestra

\begin{tabular}{|l|c|c|c|c|c|c|}
\hline ENTEROBACTERIAS & BUCO-NASAL & $\%$ & BRANQUIAL & $\%$ & TOTAL & $\%$ \\
\hline Escherichia coli & 8 & $31 \%$ & 4 & $15 \%$ & 12 & $46 \%$ \\
\hline Klebsiella oxitoca & 1 & $4 \%$ & 0 & $0 \%$ & 1 & $4 \%$ \\
\hline Negativo & 9 & $35 \%$ & 3 & $12 \%$ & 12 & $46 \%$ \\
\hline Proteus mirabilis & 1 & $4 \%$ & 0 & $0 \%$ & 1 & $4 \%$ \\
\hline Total general & 19 & $73 \%$ & 7 & $27 \%$ & 26 & $100 \%$ \\
\hline
\end{tabular}

La prevalencia de enterobacterias en tilapias en el presente estudio oscila del 4 al $46 \%$ y al comparar con datos estadísticos de otros países: Colombia (35\%) (Corrales, 2011), y Costa Rica del 50 al 90\% (Neyts, 2000; González, 2001), se puede considerar que la infección por enterobacterias en las muestras analizadas se encuentra entre los parámetros registrados en Latinoamérica y pueden representar un alto riesgo para la salud pública.

Por otro lado, en Colombia durante el 2006, se identificó Proteus mirabilis (20\%) en tilapia roja y carne en descomposición, además, su proliferación tiene una fuerte asociación con las moscas encontradas en los mercados de expendios, las mismas que son una de las fuentes de contaminación con Enterobacterias en los alimentos. Asimismo, existe mayor predominio de este género en el intestino de las moscas (Corrales, 2011).Es importante destacar que en nuestro país no existen datos sobre el referente, por lo que constituye un gran aporte para la piscicultura y salud pública

\section{CONCLUSIONES}

Se realizó un estudio bacteriológico de enterobacterias, en tilapias comercializadas en el cantón Milagro, durante los meses de octubre y noviembre del 2013, resultando una prevalencia entre 4 al 46\%. Se identificó Escherichia coli, Proteus mirabilis y Klebsiella oxitoca en tilapias por los métodos microbiológicos estandarizados, relacionándose con otros trabajos de investigación en los que se detectó Escherichia coli, Klebsiella oxitoca, Citrobacter spp., Salmonella spp., Proteus spp., Vibrio spp., Enterobacter spp. Shigella, entre otras (Puerta-García, 2010); El nivel de contaminación por enterobacterias en tilapias se relaciona con estudios realizados en otras investigaciones a nivel mundial y pueden transmitirse a humanos, lo cual constituye un riesgo para los acuicultores y consumidores que puede conllevar a una morbimortalidad.

\section{RECOMENDACIONES}

Utilizar medidas de control sanitario durante la etapa de producción y comercialización, concientizar a los productores y expendedores del uso adecuado de las normas sanitarias para el expendio de tilapias, realizar estu- 
dios en otras zonas con un mayor número de muestras, en investigaciones posteriores, confirmar las muestras aisladas por Biología molecular y tomar medidas preventivas en la cocción y manipulación de la tilapia.

\section{REFERENCIAS BIBLIOGRÁFICAS}

Betty A. Forbes, D. S. (2009). Bailey \& Scott. Diagnóstico Microbiológico (12 ed.). Buenos Aires Argentina: Panamericana.

CLSI. (2015). Performance Standards for Antimicrobial Susceptibility Testing; Twenty-Fifth Informational Supplement (Vols. M02-A10, (M100-S25) and M07A10.2015.). Clinical and Laboratory Standards Institute (CLSI).

Corrales Ramírez, L. A. (2011). Estudio bacteriológico de la calidad del pescado fresco , Bagre (Pseudoplatystoma sp . ) y Mojarra Roja ( Oreochromis sp .) comercializado en el municipio de El colegio, Cundinamarca ( Colombia). Nova-Publicación Científica en Ciencias Biomédicas, 9(15), 149-157.

Corrales, L. C. (2011). Estudio bacteriológico de la calidad del pescado fresco, Bagre (Pseudoplatystoma sp.) y Mojarra Roja (Oreochromis sp.) comercializado en el municipio de El Colegio, Cundinamarca.

Fernando Paredes Salido, J. J. (Mayo de 2004). Infecciones Gastrointestinales. Offarm, 23(5).

González, C. S. (2001). Mesophilic Aeromonads in wild and aquacultured freshwater fish. Journal of food protection.

Leal, Y. R. (2009). Enteropatogenicidad de bacterias aisladas de peces, del agua y plancton de su entorno en Venezuela. (Scielo, Ed.) Leal, Yubiry, Reyes, Mayra, Álvarez, Julia D, Obregón, José, \& Viña, Xiomara. (2009). EnteropatogenicidRevista Científica, 19(5), 446-454.

MacFaddin. (2004). Pruebas bioquímicas para la identificación de bacterias de importancia clínica (3ra ed.). Buenos Aires, Argetina: Panamericana.

Murray, P. R. (2009). Microbiología Médica (6ta ed.). Barcelona, España: Panamericana.

Neyts K, H. G. (2000). Letters in applied microbiology. (P. Med, Ed.) Incidence and identification of mesophilic Aeromonas spp. from retail foods., 31(5).

Puerta-García, E. M.-R. (2010). Enterobacterias. Medicina, Unidad de enfermedades Infeciosas Servicio de Medicna Interna, Hospital Universitario, 10.

Rocha R, L. L. (2014). Antimicrobial Susceptibility of Escherichia coli Isolated from Fresh-Marketed Nile Tilapia (Oreochromis niloticus). Journal of pathogens.

Winn, Allen, Janda, Koneman, Procop, Schreckenberger, \& Woods. (2008). Diagnóstico Microbiológico. Madrid: Panamericana.

Garzón, J. C. (2014). Calificar los efectos de la poca inversión de la acuacultura a pequeña escala para brindar un asesoramiento enfocado en la tilapia como crianza redituable en el cantón Milagro. Rev. Universidad estatal de milagro. Recuperado de:http://repositorio.unemi.edu.ec/bitstream/123456789/1155/3/Calificar\%20los\%20efectos.

\section{6}


